\title{
Review: behavioural interventions have a small to medium beneficial effect on diabetes management in adolescents with type 1 diabetes
}

\author{
Hampson SE, Skinner TC, Hart J, et al. Behavioral interventions for adolescents with type 1 diabetes: how effective are \\ they? Diabetes Care 2000 Sep;23:1416-22.
}

\begin{abstract}
QUESTION: In adolescents with type 1 diabetes, how effective are behavioural interventions in improving management of diabetes?
\end{abstract}

\section{Data sources}

Studies were identified by searching 11 databases to June 1999; handsearching the journals Diabetes Care, Diabetic Medicine, and Practical Diabetes International; contacting researchers in the field for published or unpublished work; and scanning reference lists for additional articles.

\section{Study selection}

Studies were selected if they were empirical studies involving participants 9-21 years of age with type 1 diabetes, and the study reported an evaluation of a behavioural intervention ( $\geqslant 1$ of education, skills training, or any other form of behavioural or psychosocial intervention designed to improve adolescents' diabetes management). The studies were also screened for randomised, non-randomised, or waiting list control group designs.

\section{Data extraction}

Data were extracted on study design, number of participants, type of intervention, and effects on outcomes. Effect sizes were calculated and outcomes were categorised as psychosocial (eg, self efficacy for diabetes management, measures of family climate or family conflict, diabetes specific stress, and quality of life), glycated haemoglobin $(\mathrm{GHb})$, other metabolic measures, self management behaviours, and knowledge of diabetes.

\section{Main results}

35 studies had randomised, non-randomised, or waiting list control group designs, and involved a mean of 55 patients (mean age $12 \mathrm{y}$, mean duration of diabetes $5 \mathrm{y}$ ). 24 studies were randomised controlled trials; 16 trials evaluated 18 interventions with sufficient data to calculate effect sizes. An overall small to medium beneficial effect of behavioural interventions on adolescent diabetes management was seen when the mean effect sizes of all studies were pooled (the table shows the mean effect sizes for each outcome). Theoretically based interventions were more effective than those that were not.
879553

National Healt

Service Health

Programme.

For correspondence:

Dr S E Hampson,

Psychology, University

of Surrey, Guildford,

Surrey GU2 $5 \mathrm{XH}, \mathrm{UK}$

\section{Conclusion}

In adolescents with type 1 diabetes, behavioural interventions have a small to medium beneficial effect on diabetes outcomes.

\section{COMMENTARY}

Clear evidence exists that strict control of blood sugars in patients with type 1 diabetes mellitus ameliorates the progression of microvascular complications. Psychosocial and behavioural factors have an important influence on the adequacy of metabolic control. Hampson et al reviewed the effectiveness of behavioural interventions for adolescents with type 1 diabetes. They found that the mean effect size for the interventions across all outcomes, and for $\mathrm{GHb}$ in particular, was 0.33 , indicating a small to medium sized beneficial effect. This effect size for $\mathrm{GHb}$ improvement translates into a change of $0.6 \%$ in haemoglobin $A_{\mathrm{lc}}$, which is about one third of that found in the Diabetes Control and Complications Trial. It is encouraging that some benefit can be shown for interventions in this age group, but the persistence of the improvement and the extent to which it lowers the risk of complications remains to be determined.

This review showed that interventions with a theoretical foundation were more effective than other interventions, with the most effective involving family interventions, coping skills training, and problem solving related to diabetes management. However, the review does not discuss interventions for specific problems such as depression or eating disorders, both of which are common among adolescents with diabetes. ${ }^{12}$ In fact, eating disorders in adolescents with diabetes have been associated with a three-fold increase in the risk of retinopathy. ${ }^{3}$

Although problem solving and skill training may be effective for a broad mix of patients, there is likely to be value in tailored interventions directed to alleviate specific disturbances. Further research is needed, however, to clarify the persistence of change and the health benefits associated with behavioural interventions in adolescents with type 1 diabetes.

Gary Rodin, MD, FRCPC Toronto General Hospital Toronto, Ontario, Canada

1 Kovacs M, Goldston D, Obrosky DS, et al. Psychiatric disorders in youth with IDDM: rates and risk factors. Diabetes Care 1997;20:36-44.

2 Jones JM, Lawson ML, Daneman D, et al. Eating disorders in adolescent females with and without type 1 diabetes: cross sectional study. BMJ 2000;320:1563-6.

3 Rydall AC, Rodin GM, Olmsted MP, et al. Disordered eating behavior and microvascular complications in young women with insulin-dependent diabetes mellitus. $N$ Engl J Med 1997;336:1849-54 